\title{
Toolkit for simulation modeling of queue systems in Grid
}

\author{
A G Feoktistov ${ }^{1}$, R O Kostromin ${ }^{1}$, E S Fereferov ${ }^{1}$, A Tchernykh ${ }^{2,3,4}$, \\ O Yu Basharina ${ }^{5}$, V I Dmitriev ${ }^{5}$ and Ya V Kurzybova ${ }^{5}$ \\ ${ }^{1}$ Matrosov Institute for System Dynamics and Control Theory of SB RAS, \\ Lermontov St. 134, Irkutsk, Russia, 664033 \\ ${ }^{2}$ CICESE Research Center, Ensenada-Tijuana Highway, Ensenada, Mexico, 22860, \\ 3918 \\ ${ }^{3}$ Ivannikov Institute for System Programming of RAS, Alexander Solzhenitsyn st. 25, \\ Moscow, Russia, 109004 \\ ${ }^{4}$ South Ural State University, Lenin prospect 76, Chelyabinsk, Russia, 454080 \\ ${ }^{5}$ Irkutsk State University, Karl Marx St. 1, Irkutsk, Russia, 664003 \\ agf@icc.ru
}

\begin{abstract}
The paper addresses an important problem related to automation of simulation modeling of queue systems using high-performance computing. There is a large spectrum of tools for the simulation modeling of such systems. However, these tools often do not use the possibilities of subject-oriented high-performance computing in full. The purpose of the study is the development of the new tools for automation of the simulation modeling of queue systems in a heterogeneous distributed computing environment. To this end, we develop specialized applications for parameter sweep computations in such an environment. A methodology of the application development is based on the integrated use of methods of the conceptual and wireframe programming, methods of the computations planning and resources allocation, methods of parameter sweep computations, and methods of the data analysis. We develop the toolkit named SIRIUS III for the simulation modeling of queue systems using high-performance computing. In addition, we design a conceptual model that represents knowledge about both the subject domain of solved problems and computing environment. Using the proposed toolkit, we solved a number of problems related to warehouse logistics. Computational experiments showed the high scalability of parameter sweep computations on heterogeneous resources.
\end{abstract}

\section{Introduction}

Nowadays, the study of the functioning of complex technical and economic queue systems at various stages of their design, testing and operation is one of the important problems in the field of simulation modeling [1].

Simulation modeling simplifies the development of optimal schemes for the operation of queue systems and the selection of their control parameters [2]. Often, the simulation results quality directly depends on the use of knowledge about the subject domain specifics of the queue system under study in the problem-solving process $[3,4]$.

The process of developing a simulation model that adequately reflects the queue system under study is a complex problem. It requires a model developer to have high mathematical and programmer qualifications. Especially, when the model is studied using high-performance computing. 
In this regard, there is a need to create specialized high-level tools to support this process. Firstly, these tools will allow use at maximum the potential of high-performance computing. Secondly, they will provide automation of the process of the development and applying a simulation model at all levels. This process begins from a mathematician (who formalize of the subject domain of the queue systems), to a programmer (who creates a simulation model of the queue systems that is oriented to Grid), and, finally, to end-users (who directly carry out parameter sweep computations).

In the paper, we propose the specialized SIRIUS III toolkit to support simulation modeling of queue systems based on parameter sweep computations in Grid. The parameter sweep application that is developed in SIRIUS III generates a large number of independent jobs for the computing environment.

The job determines the problem-solving process (model run). It describes the required resources, executable program (model), input and observed variables of a model, information about resource virtualization options, etc. This description is formed by users or generated by their applications automatically. Then, the job is submitted to local resource managers or meta-schedulers located in computational nodes of Grid.

Each job runs the model with a variant of values of its input variables. In the parameter sweep application, we apply multi-criteria methods to select a problem solution based on the variants of observed variables of the model.

The rest of the paper is structured as follows: in Section 2, we give a brief overview of the known tools for simulation modeling of queue systems and propose a new methodology for simulation modeling such systems in Grid. Section 3 describes the SIRIUS III toolkit used for simulation modeling of queue systems in Grid. Computational experiments related to solving warehouse logistics problems are represented in Section 4. Finally, Section 5 concludes the paper.

\section{Related work}

An analysis of the state of the art in the field of simulation modeling of queue systems shows that the spectrum of tools for simulation modeling is very large [5-8]. However, often, these tools do not use high-performance computations [9] or do not provide a consideration of subject domain specifics for solved problems $[10,11]$.

Parallel and distributed computing provides the following significant advantages [12]:

- Large-scale simulation experiments,

- Ability to generate and use big input data,

- Range extension of problems of the prediction, management, and risk analysis that are solved in the decision-making process.

At the same time, it is necessary to note the modern trend related to the integration of simulation and optimization methods [13].

Given the advantages and drawbacks of well-known simulation tools, we propose the simulation modeling methodology that includes the following stages:

- Development of the conceptual model of the studied queue system in terms of a subject domain using the methods of conceptual programming,

- Automated creation of the simulation model of the studied queue system based on the conceptual model (the development of the parameter sweep application) using the wireframe approach to the program design,

- Configuring resources of the heterogeneous distributed computing environment (Grid) for the application execution,

- Carrying out experiments with the simulation model (parameter sweep application) in the heterogeneous distributed computing environment (Grid),

- Analyzing the simulation results using multi-criteria and statistical methods.

Implementation of this methodology is based on the SIRIUS III toolkit use. Simulation models of queue systems are generated in the GPSS language [14]. We can note that the simulation environment GPSS World is constantly evolving [15]. 


\section{The SIRIUS III toolkit}

We developed the SIRIUS III toolkit to automate the creation of simulation models based on templates (modules) of typical objects of the studied systems. In addition, SIRIUS III is applied to prepare and carry out computational experiments with the created simulation models. The models are implemented in the GPSS language terms. Simulation models of queue systems are created using a modular approach that provides a number of advantages.

The modular approach allows us to study various versions of the simulated system by painlessly adding or replacing modules that reflect the mathematical and software basis of the algorithmic knowledge. The modules are represented as templates of typical objects of the simulated system. They are stored in a pre-formed library. SIRIUS III provides quick and pointwise implementation of additional possibilities of modeling the queue system that are not represented in the means used to control this system in practice. Auxiliary analytical models can be implemented in SIRIUS III as modules.

The methodology for constructing a conceptual model of the system under study is based on the general principles of structural analysis of the subject domain. Among them, we highlight identifying objects of a subject domain that interact with each other, determining their main attributes, defining relations between objects and restrictions on these relations. The conceptual model is the basis for creating simulation models.

The architecture of the SIRIUS III toolkit includes the web-interface, conceptual model designer, knowledge base, simulation model designer, executive subsystem, computations database, and analysis subsystem (figure 1). In addition, SIRIUS III uses the external GeoARM System and tools for continuous integration, delivery, and deployment of software.

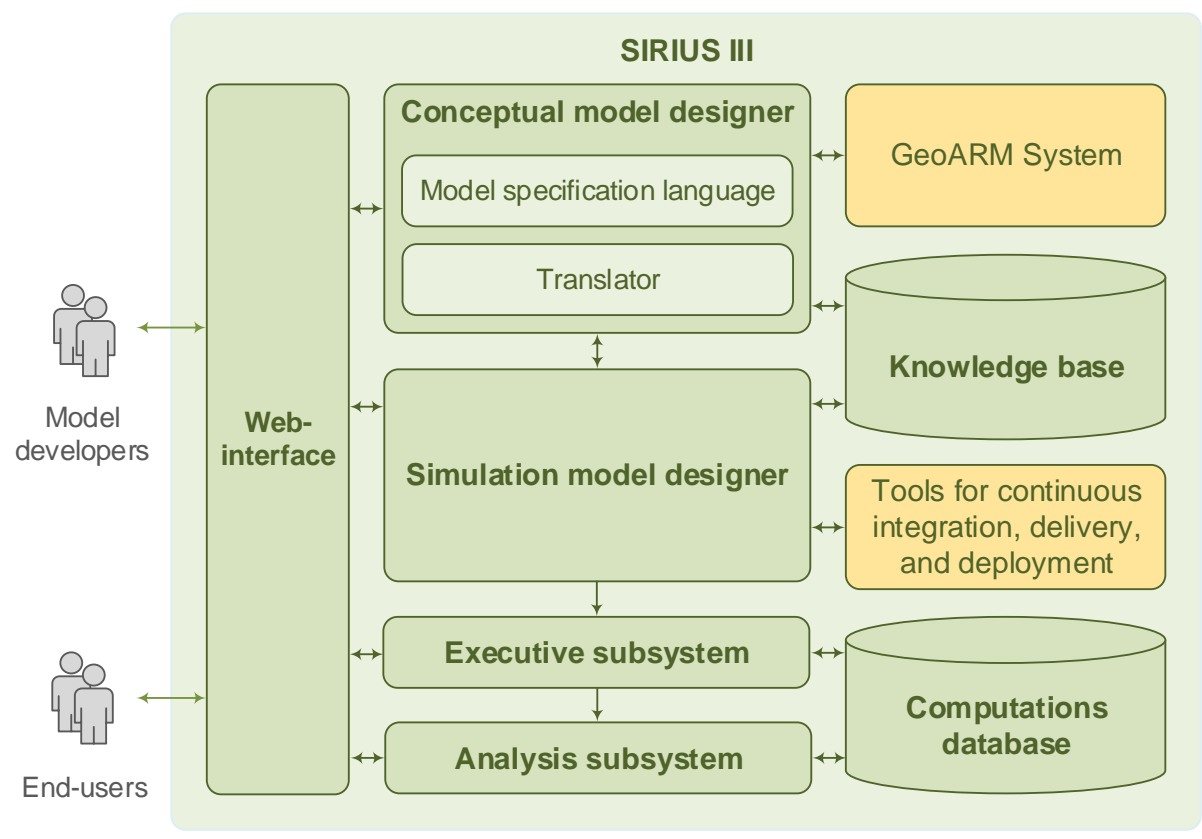

Figure 1. Architecture of the SIRIUS III toolkit.

The conceptual model designer provides developers with the opportunity to describe the queue system model in a special model specification language. This language is an ontological language. It enables to describe the objects (concepts) of the studied subject domain, set their attributes, determine the data types of the attributes, define relations between the objects, and impose restrictions on these relations. The use of the model specification language provides a high degree of adequacy of subject domain reflection by the conceptual model due to the ability to create a variety of subject domain concepts. 
The conceptual model includes the following knowledge components:

- Computational knowledge about software modules for solving problems in subject domains and operating with queue system objects,

- Schematic knowledge about the modular structure of the model and algorithms,

- Production knowledge to support decision-making on the selection of optimal algorithms depending on the computing environment state,

- Knowledge about the hardware and software infrastructure of the computing environment,

- Information about administrative quotas and policies at computing environment nodes.

A translator transforms the conceptual model description into the SQL language terms. Then, a knowledge base is created. Its structure is determined using the GeoARM system [16]. This system provides model developers with an opportunity to automatically generate a unique graphical interface for each specialized model. This interface is used further for filling the knowledge base by model developers and end-users. Conceptual models descriptions are stored in the knowledge base and can be used to create new models.

We create the simulation model using the GPSS language based on the wireframe approach. Within the wireframe approach, a model consists of two mandatory parts: a wireframe (skeleton structure) and slots. The wireframe is a part of the GPSS model, which does not change with the model versions change. It includes a set of slots (a variable part of the model) for plug-in modules that are placed into slots. Plug-in modules are parts of computational knowledge. The set of modules is a library of fragments of the simulation model. All the diversity of the generated versions of the model is achieved by changing the contents of slots in different versions.

Supporting the module versions use that are created by different developers is implemented through applying tools of continuous integration, delivery, and deployment of software. This significantly increases the reliability of computations in carrying out further experiments with the simulation model.

The model wireframe and its slots are created in the simulation model designer (figure 2).

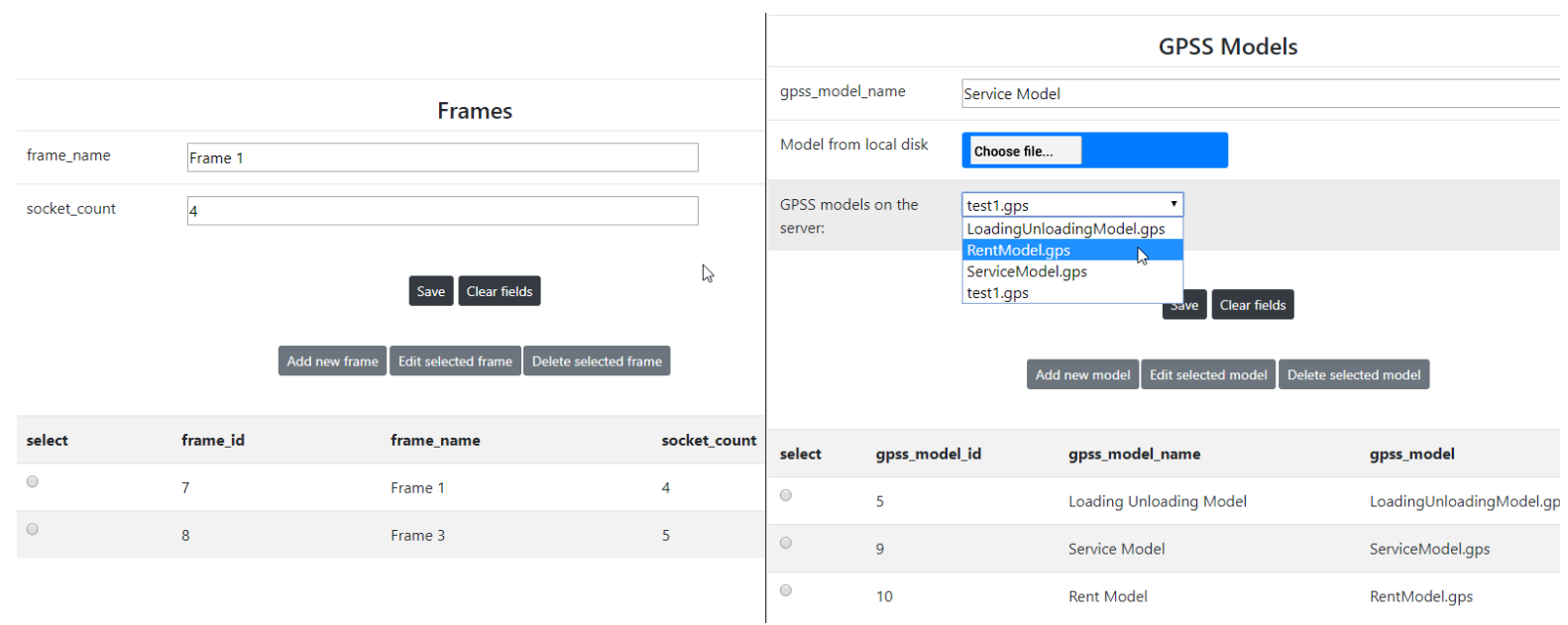

Figure 2. Simulation model designer.

The executive subsystem provides the following features:

- Access to computational resources,

- Formulating problem statements

- Preparation of variants of initial data,

- Generating jobs,

- Managing parameter sweep computations,

- Organization of interaction with the task control system of the computing cluster, 
- Carrying out simulation modeling,

- Obtaining simulation results and their post-processing based on the methods of statistical analysis and multi-criteria optimization.

GPSS World is launched in the computing environment using virtual machines that are running under specialized hypervisors (VMware, Xen or KVM).

The computations database keeps information about problem formulations, problem-solving schemes, and parameter values.

The analysis subsystem selects the optimal model parameters using the following methods of multicriteria selection: lexicographic, majority and Pareto-optimal.

\section{Computational experiments}

We apply SIRIUS III to solve a number of problems that are associated with the study of warehouse logistics processes. Traditional warehouse management systems do not support the solving of these problems [17]. Profit, filling the warehouse, maintenance costs, resource efficiency, and other parameters are criteria of warehouse functioning effectiveness.

Problem 1: Loading and unloading goods. The studied warehouse performs operations with input and output material flows. We consider flows with both the planned and random requests. Planned requests are formed every day. They contain information about the time of the arrival and departure of a cargo, request service time, cargo volume, and required resources for servicing. The following technical and human resources are used for servicing: electric loader, drivers of electric loaders, loaders and storekeepers. It is necessary to study and compare different variants of loading and unloading goods.

Problem 2: Service level for loading and unloading goods. The warehouse provides services for loading and unloading goods using road and rail transport of various carrying. There are standards for such operations taking into account the cargo transport type and cargo category. Failure to comply with these regulations entails a fine. It is necessary to determine the number of warehouse resources that will provide the following restrictions:

- Average waiting time of road and rail transport at loading and unloading goods is less than the specified value,

- Idle time of warehouse personnel do not exceed the specified value,

- Parameters of services for loading and unloading goods remain within acceptable limits.

Problem 3: Renting out the commercial real estate. The warehouse rent out the unused commercial real estate. Each object belongs to one of the categories (warehouse, garage, office, etc.). Warehouse management determines the possibility (or impossibility) of its re-equipment for transfer from one category to another. In addition, warehouse management set the price of renting objects, cost of their maintenance, and probable demand for rental objects in different periods of the year. It is necessary to determine the plan of delivery of objects for renting and rental period taking into account the operation and maintenance of commercial real estate in the overall process of warehousing.

We developed three simulation models for solving the aforementioned problems.

The model adequacy is evaluated by verification and validation methods. The model verification provides evaluating the correctness of the conceptual model translation to the simulation program. The correctness of a model behavior logic is verified through the following actions:

- Interactive controlling the simulation process using the debug utilities of GPSS,

- Analyzing the simulation results correctness for "extreme" values (zero and non-existent values of input parameters of the model),

- Comparing the simulation results with the values obtained by analytical calculation.

Validation methods are used to verify the facts that the simulation results have a satisfactory accuracy and do not conflict with the system under study. Validation is performed by comparing the responses of the real system and its simulation model.

Figure 3(a) shows the standard deviation of the simulation results from the real system parameters for the main logistics operations by months. The obtained deviation demonstrates the simulation results stability. Figure 3(b) shows the averaged standard deviation of the simulation results for the main 
logistics operations from the real system parameters. The deviation does not exceed $8.03 \%$. Thus, we can conclude that the simulation model is a valid model.

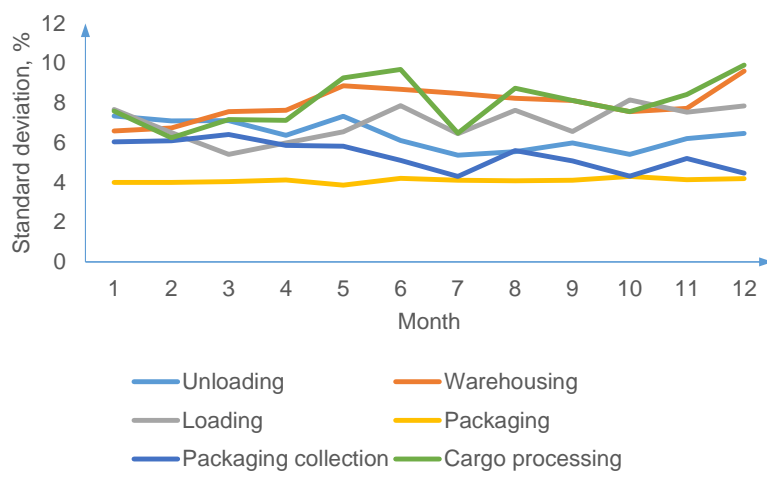

a)

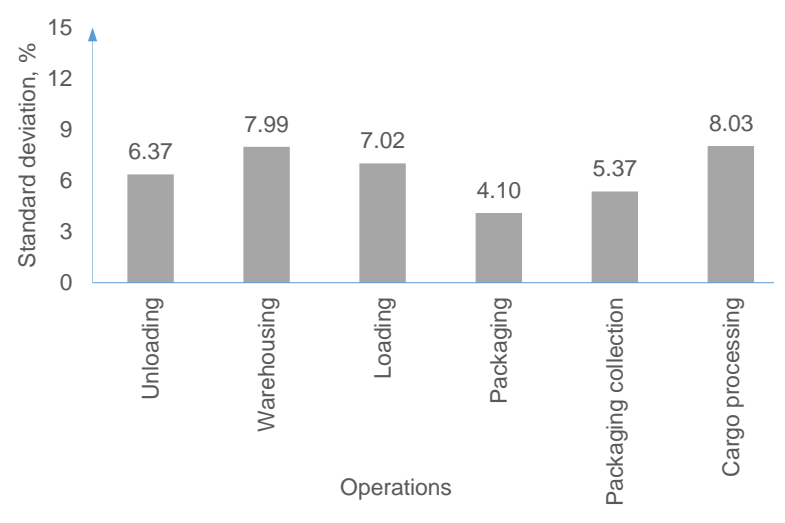

b)

Figure 3. The standard deviation of the simulation results of the main logistics operations from the real system parameters by months (a) and their average standard deviation (b).

Problems were solved by parameter sweep computations. The multi-criteria selection methods were used to select the simulation model optimal parameters. Values of the observed variable in the models matched the specified criteria for the quality of operation and performance of the warehouse. A special feature of solving these problems is taking into account failures of technical means and risks associated with human factors. The planning of experiments was carried out in accordance with the proposed methodology.

The results obtained in solving problem 1 and problem 2 are used by warehouse managers in the following decision-making:

- Forming the service schedule of planned customer requests and accounting for random customer requests,

- Scheduling teams of loaders and storekeepers,

- Creating plans of both the warehouse maintenance and updating electric loaders,

- Setting standards for loading and unloading goods,

- Determining categories of «loyal» customers.

These results allowed improving the eight criteria of a warehouse functioning by from $6 \%$ to $75 \%$. The following criteria were improved: loaders efficiency, average time of the loading and unloading operations, average profit of the loading and unloading operations, average time of the packaging collection, profit of the packaging, storekeepers number, elevators calls, and warehouse filling.

The results obtained in solving problem 3 made it possible to increase the planned financial parameters of warehousing by $15 \%$.

The experiments were carried out in the Grid that integrates heterogeneous resources of the public access supercomputer center "Irkutsk Supercomputer Center" [18]. We use three pools of resources:

1) Ten computational nodes with 2 processors AMD Opteron 6276 (16 core, $2.3 \mathrm{GHz}, 64 \mathrm{~GB}$ of RAM) for each,

2) Ten computational nodes with 2 processors Intel Xeon CPU X5670 (18 core, $2.1 \mathrm{GHz}, 128 \mathrm{~GB}$ of RAM) for each,

3) Five computational nodes of the pool 1 and five nodes of the pool 2.

Figure 4(a) and figure 4(b) correspondingly show the computations speedup and resources use efficiency achieved in solving problems 1-3 on a various number of computational nodes in the pool 1 . A number of cores in the pool 1 is changed from 32 to 320 . Obviously, the computations speedup close to the linear speedup and resources use efficiency close to the efficiency equal to one for all problems. 


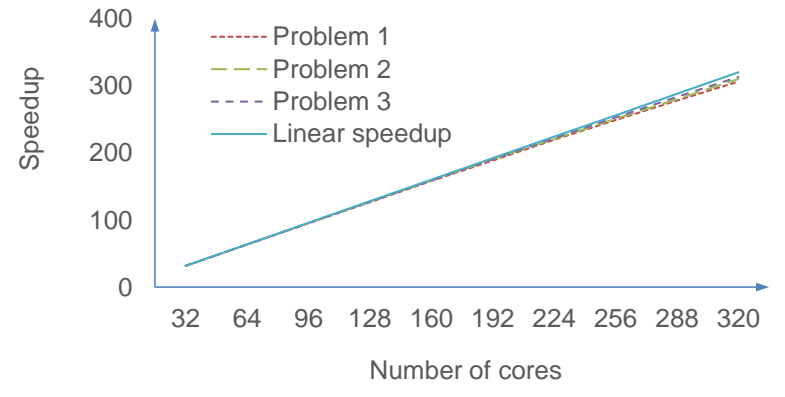

a)

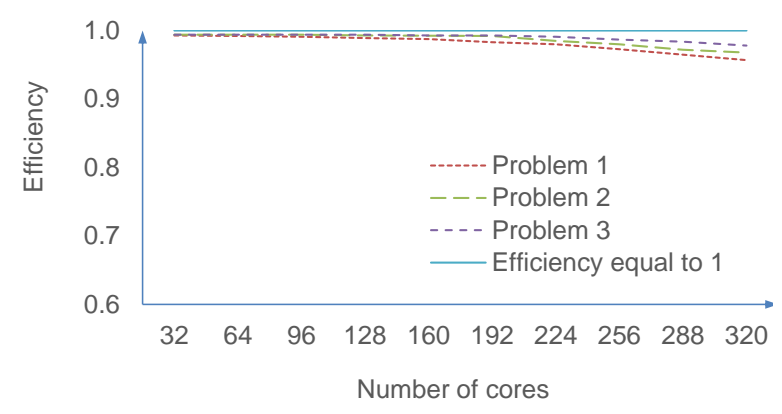

b)

Figure 4. Computations speedup (a) and resources use efficiency (b) with computational nodes of the pool 1.

Figure 5(a) and figure 5(b) correspondingly show the computations speedup and resources use efficiency achieved in solving problems 1-3 on a various computational number of nodes in the pool 2. A number of cores in the pool 2 is changed from 36 to 360 . We can see that the computations speedup and resources use efficiency in both cases (figure 4 and figure 5) about equal.

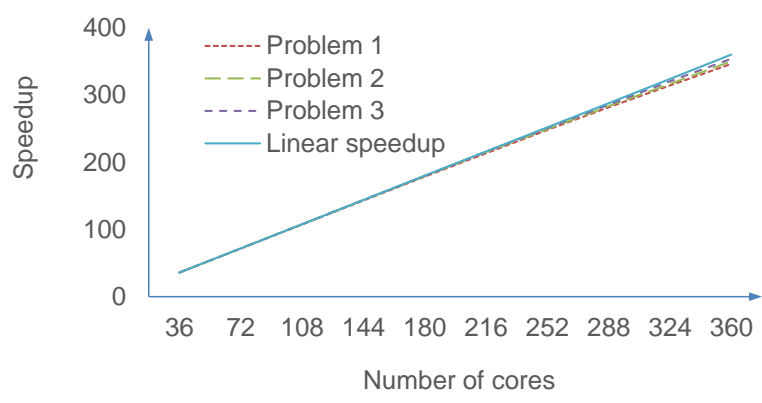

a)

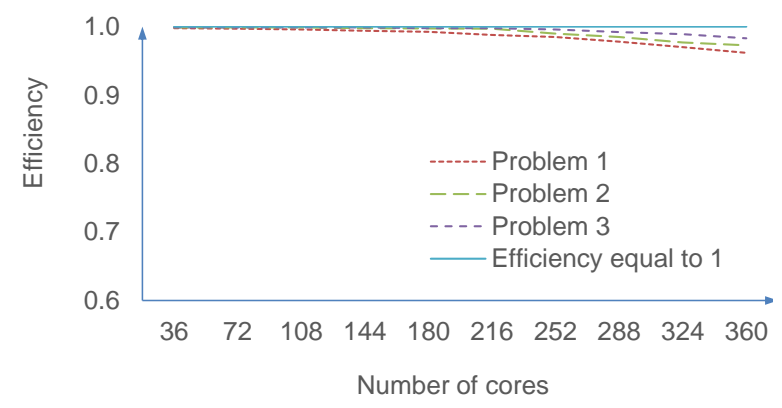

b)

Figure 5. Computations speedup (a) and resources use efficiency (b) with computational nodes of the pool 2.

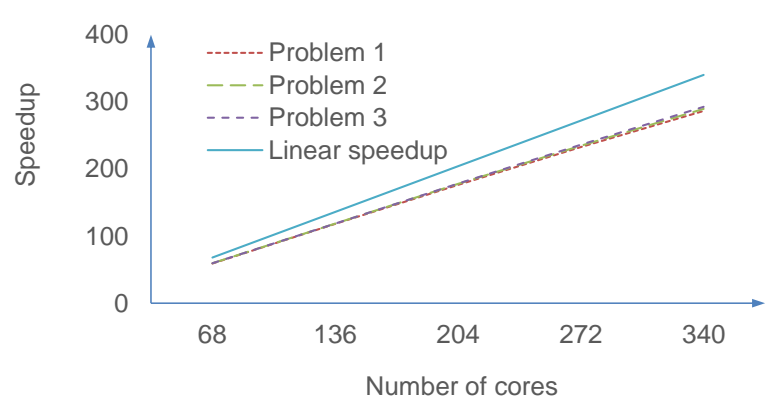

a)

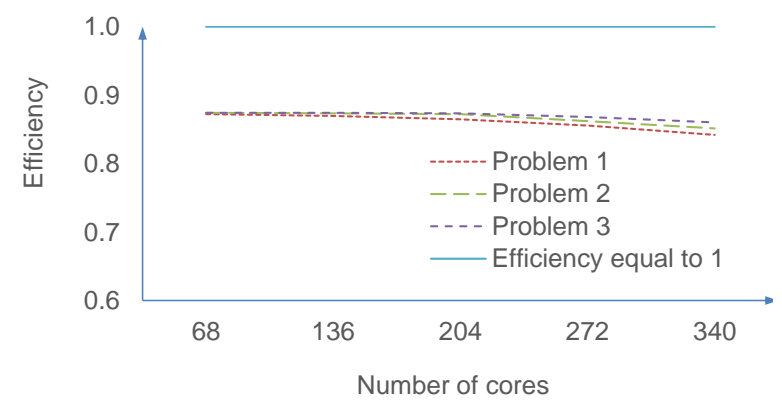

b)

Figure 6. Computations speedup (a) and resources use efficiency (b) with computational nodes of the pool 3.

When we apply computational nodes of the pool 3, the computations speedup and resources use efficiency decreases slightly (figure 6) in comparison with computations in the pool 1 (figure 4) and 
pool 2 (figure 5). This is owing to the resource heterogeneity in the pool 3. The speedup and efficiency are calculated relatively the problem solving time on one core of the most high-performance computational node of the pool 2. At the same time, the problem-solving time is significantly reduced in comparison with the problem-solving time on one core. The average processor load is high for nodes of both types in the pool 3 (figure 7). Thus, we can conclude that computational experiments showed the high scalability of parameter sweep computations on heterogeneous resources.

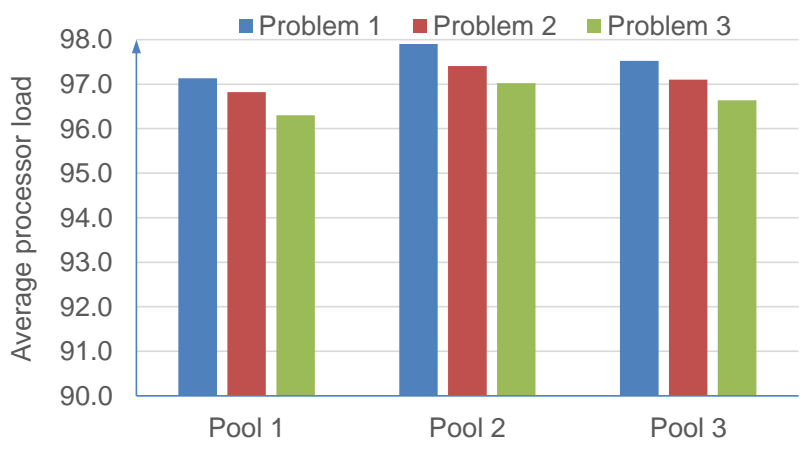

Figure 7. Average processor load.

Additional aspects of solving warehouse logistics problems in the heterogeneous distributed computing environment that are related to the multi-agent management of distributed computing and multi-criteria optimization of the modeling results are discussed in detail in [19, 20].

\section{Conclusions}

In the paper, the problem related to the automation of simulation modeling of queue systems using highperformance computing is considered. Often, well-known tools for simulation modeling of queue systems do not use high-performance computations or do not consider subject domain specifics for solved problems. In this regards, we propose a new approach to simulation modeling of queue systems based on parameter sweep computations in Grid. Within the proposed approach, we highlight a number of advantages.

SIRIUS III provides the opportunity to take into account the subject domains specifics in solving problems. We apply modular structure of simulation models and automate their construction based on the wireframe approach. The collaborative work of different users (model developers and end-users) with modules are coordinated through continuous integration. We support parameter sweep computations using high-performance computing and multi-criteria optimization of modeling results.

Computational experiments show the high scalability of distributed computing and effectiveness in using Grid resources.

Acknowledgment. The study is supported by the Russian Foundation of Basic Research, projects no. 19-07-00097-a (reg. no. AAAA-A19-119062590002-7) and no. 18-07-01224. This work was also supported in part by Basic Research Program of SB RAS, project no. IV.38.1.1 (reg. no. AAAA-A17117032210078-4).

\section{References}

[1] Taha H A 2016 Operations research: an introduction (Cambridge: Pearson) p 848

[2] Bruzzone A and Longo F 2014 An Application Methodology for Logistics and Transportation Scenarios Analysis and Comparison within the Retail Supply Chain Eur. J. Ind. Eng. 18(1) $112-142$

[3] Robinson S 2013 Conceptual Modeling for Simulation Proc. of the 2014 Winter Simulation Conf. (Piscataway: IEEE Press) pp 377-388 
[4] Felice F D and Petrillo A 2015 Optimization of Manufacturing System through World Class Manufacturing IFAC-PapersOnLine 48(3) 741-746

[5] Sulistio A 2014 A Taxonomy of computer-based simulations and its mapping to parallel and distributed systems simulation tools Software Pract. Exper. 34(7) 653-673

[6] Schriber T J, Brunner D Tand Smith J S 2014 Inside discrete-event simulation software: how it works and why it matters Proc. of the 2014 Winter Simulation Conf. (Piscataway: IEEE Press) pp 132-146

[7] Macal C and North M 2014 Introductory tutorial: agent-based modeling and simulation Proc. of the 2014 Winter Simulation Conf. (Piscataway: IEEE Press) pp 6-20

[8] Dias Luis M S, Vieira Antonio A C, Pereira Guilherme A B and Oliveira Jose A 2016 Discrete simulation software ranking - a top list of the worldwide most popular and used tools Proc. of the 2016 Winter Simulation Conf. (Piscataway: IEEE Press) pp 1060-1071

[9] Borshchev A, Karpov Y and Kharitonov V 2002 Distributed simulation of hybrid systems with AnyLogic and HLA Future Gener. Comp. Sy. 18(6) 829-839

[10] Fujimoto R 2015 Parallel and distributed simulation Proc. of the 2015 Winter Simulation Conf. (Piscataway: IEEE Press) pp 45-59

[11] Beloglazov A, Banerjee D, Hartman A and Buyya R 2015 Improving productivity in design and development of information technology (IT) service delivery simulation models J. Serv. ResUS 18(1) 75-89

[12] Nelson B L 2016 Some Tactical Problems in Digital Simulation for the Next 10 Years J. Simul. $102-11$

[13] Cheng R, Macal C, Nelson B, Rabe M, Currie C, Fowler J and Lee L H 2016 Simulation: the past 10 years and the next 10 years Proc. of the 2016 Winter Simulation Conf. (IEEE Press) pp 2180-2192

[14] Schriber T J 1974 Simulation using GPSS (New York: John Wiley)

[15] Vlasov S A, Deviatkov V V, Isaev F V and Fedotov M V 2014 Imitational Studies with GPSS WORLD: New Capabilities Automat. Rem. Contr.+ 75(2) 389-398

[16] Fereferov E S, Bychkov I V and Khmel'nov A E 2014 Technology for Database Applications Based on Declarative Specifications Journal of Computational Technologies 19(5) 85-100 (In Russian)

[17] Gwynne R 2014 Warehouse Management: a Complete Guide to Improving Efficiency and Minimizing Costs in the Modern Warehouse (London: Kogan Page) p 448

[18] http://hpc.icc.ru

[19] Bychkov I, Oparin G, Tchernykh A, Feoktistov A, Bogdanova V, Dyadkin Yu, Andrukhova V and Basharina O 2017 Simulation Modeling in Heterogeneous Distributed Computing Environments to Support Decisions Making in Warehouse Logistics Procedia Engineering 201 pp 524-533

[20] Bychkov I, Feoktistov A, Sidorov I and Kostromin R 2017 Job Flow Management for Virtualized Resources of Heterogeneous Distributed Computing Environment Procedia Engineering 201 pp 534-542 